\title{
Excited-states and spectroscopic properties of superoxide anion: a theoretical study including spin-orbit coupling
}

Hui Liu ${ }^{\text {a, b }}$, Deheng Shi ${ }^{\text {a,* }}$, Jingfeng Sun ${ }^{\text {a,* }}$, and Zunlue Zhu ${ }^{\mathrm{a}}$

${ }^{a}$ College of Physics and Material Science, Henan Normal University, Xinxiang 453007, China

${ }^{b}$ College of Physics and Electronic Engineering, Xinyang Normal University, Xinyang 464000, China

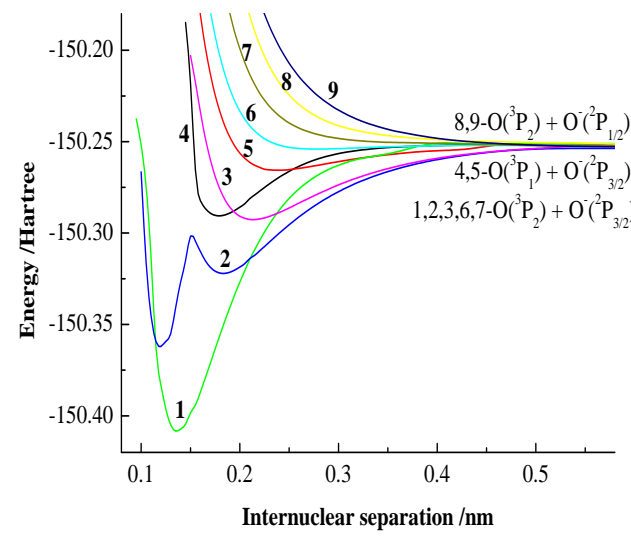

Figure I. PECs of $9 \Omega=3 / 2$ states of $\mathrm{O}_{2}{ }^{-}$anion.

$$
\begin{aligned}
& 1-\mathrm{X}^{2} \Pi_{\mathrm{g}, 3 / 2}, 2-\mathrm{a}^{4} \Sigma_{\mathrm{u}, 3 / 2}^{-}, 3-1^{4} \Sigma_{\mathrm{g}, 3 / 2}^{+}, 4-\mathrm{A}^{2} \Pi_{\mathrm{u}, 3 / 2}, \\
& 5-1^{4} \Pi_{\mathrm{u}, 3 / 2}, 6-1^{4} \Sigma_{\mathrm{g}, 3 / 2}^{-}, 7-2^{4} \Sigma_{\mathrm{u}, 3 / 2}^{-}, 8-2^{4} \Pi_{\mathrm{g}, 3 / 2}, 9-2^{4} \Sigma_{\mathrm{g}, 3 / 2}^{-} .
\end{aligned}
$$

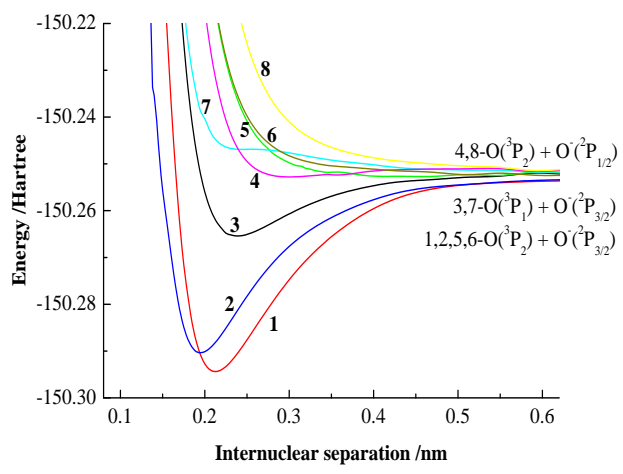

Figure III. PECs of $8 \Omega=5 / 2$ states of $\mathrm{O}_{2}{ }^{-}$anion.

$1-1^{4} \Delta_{\mathrm{g}, 5 / 2}, 2-1^{2} \Delta_{\mathrm{u}, 5 / 2}, 3-1^{4} \Pi_{\mathrm{u}, 5 / 2}, 4-2^{4} \Pi_{\mathrm{u}, 5 / 2}$,

$5-1^{4} \Pi_{\mathrm{g}, 5 / 2}, 6-1^{4} \Delta_{\mathrm{u}, 5 / 2}, 7-1^{2} \Delta_{\mathrm{g}, 5 / 2}, 8-2^{4} \Pi_{\mathrm{g}, 5 / 2}$.

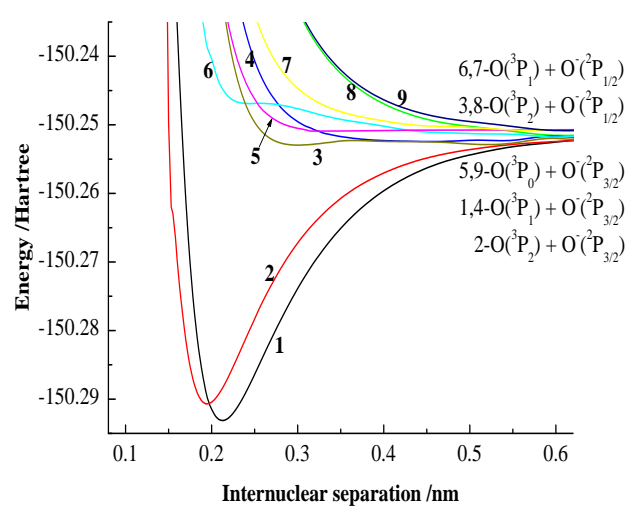

Figure II. PECs of $9 \Omega=3 / 2$ states of $\mathrm{O}_{2}^{-}$anion.

$$
1-1^{4} \Delta_{\mathrm{g}, 3 / 2}, 2-1^{2} \Delta_{\mathrm{u}, 3 / 2}, 3-1^{4} \Sigma_{\mathrm{u}}{ }^{+}, 3 / 2,4-1^{4} \Pi_{\mathrm{g}, 3 / 2}, 5-1^{2} \Delta_{\mathrm{g}, 3 / 2},
$$

$6-2^{2} \Pi_{\mathrm{g}, 3 / 2}, 7-2^{2} \Pi_{\mathrm{u}, 3 / 2}, 8-2^{4} \Pi_{\mathrm{u}, 3 / 2}, 9-1^{4} \Delta_{\mathrm{u}, 3 / 2}$

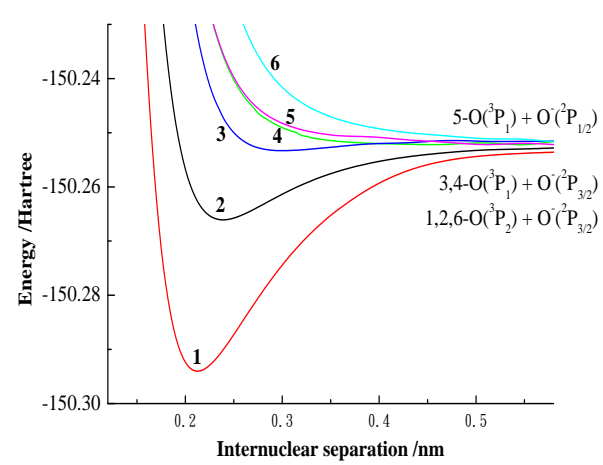

Figure IV. PECs of $4 \Omega=-1 / 2$ and $2 \Omega=7 / 2$ states of

$\mathrm{O}_{2}{ }^{-}$anion. $1-1^{4} \Delta_{\mathrm{g}, 7 / 2}, 2-1^{4} \Pi_{\mathrm{u},-1 / 2}, 3-1^{4} \Pi_{\mathrm{g},-1 / 2}$,

$4-2^{4} \Pi_{\mathrm{u},-1 / 2}, 5-2^{4} \Pi_{\mathrm{g},-1 / 2}, 6-1^{4} \Delta_{\mathrm{u}, 7 / 2}$.

*Corresponding author. Tel/fax: 86-373-3328876. E-mail: scattering@ sina.com.cn; dh_shi@htu.cn. 